\title{
Jörn Peter Hiekel / Christian Utz (Hg.), Lexikon Neue Musik, Stuttgart: Metzler / Kassel: Bärenreiter 2016
}

Schlagworte/Keywords: 20. und 21. Jahrhundert; 20th and 21st century; global history; Globalgeschichte; musical historiography; Musikhistoriographie; neue Musik; new music

Bei manchen Büchern zeigt sich erst, wenn es sie gibt, wie groß die Lücke ist, die sie füllen wollen. Unweigerlich trifft das wohl auch auf ein Handbuch der zeitgenössischen Musik zu, das sich vornimmt, die mittlerweile gut zwanzig Jahre alten Beiträge im Sachteil der Musik in Geschichte und Gegenwart "präzisierend, vertiefend und aktualisierend mit Blick auf einen ihrer besonders in Wandlung begriffenen Teilbereiche fortzuführen« (IX). Das Unternehmen zielt von vornherein nicht auf eine Supplementierung der Enzyklopädie, sondern neben der Ausweitung des Gegenstandsbereichs auf eine Umakzentuierung der Darstellungsparameter. Erklärtermaßen soll es darum gehen, der faktischen Vielfalt in der Musik des 20. und 21. Jahrhunderts gerecht zu werden und die Pluralität ihrer »Tendenzen, Kriterien, Phänomene[ ] und Intentionen « (IX), ihrer Widersprüche und Konvergenzen darzustellen. Das sei bislang nicht oder nicht genügend geschehen, so die Herausgeber in ihrer Einleitung. Stattdessen sehen sie nach wie vor ein lineares Geschichtsmodell dominieren, das seine Simplifizierungen auf historiographische Stereotype von Materialfortschritt, Teleologie und unausgesetzter Innovation stützt (XI). Ob das in dieser Pauschalität zutrifft, sei dahingestellt schaut man innerhalb des vielstimmigen Diskurses über die zeitgenössische Musik an den richtigen Stellen, dann finden sich leicht auch noch Spielarten einer solchen, ansonsten zu Recht für obsolet erklärten »sldeologie` der neuen Musik« (X). Für das vorliegende Handbuch erfüllt sie jedenfalls ihre Funktion als Abstoßpunkt, als motivierendes Gegenbild für das eigene ambitionierte Unterfangen.

Das ist legitim, umso mehr, als es die Frage nach den Gründen solcher Verengungen aufwirft. Für die Herausgeber liegen sie zum einen in der »Tendenz zur weitgehenden Ausklammerung oder Marginalisierung von weiten Tei- len der neuen Musik« in der akademischen Musikwissenschaft, Musikpädagogik, Musiktheorie und Instrumentalausbildung (XI) - eine arg pauschale Diagnose, die zumindest den Autor*innen des Bandes, von denen die weitaus meisten an Universitäten und Musikhochschulen lehren, Unrecht tut. Zum anderen verweisen die Herausgeber auf den lange dominierenden journalistisch-feuilletonistischen Diskurs über die neue Musik und dessen mangelnde Distanz zum Gegenstand, resultierend in Affirmation und Beschränktheit des Urteils: "Die oft dokumentierte Beobachtung freilich, dass journalistische oder halbwissenschaftliche Texte über neue Musik im Wesentlichen bloß das paraphrasieren, was Komponistinnen und Komponisten selbst über ihre Werke und Ideen äußerten, deutet auf einen nicht unwesentlichen Faktor für das Entstehen mancher Klischees und Einseitigkeiten vor dem Hintergrund einer kontinuierlichen Nachwirkung komponistenzentrierter Genieästhetik auch im Bereich der neuen Musik« (XII). Wie die wattierte Vorsicht der Formulierung zeigt, rührt das an einen neuralgischen Punkt: Gerade in der vergleichsweise überschaubaren und dicht vernetzten Szene der zeitgenössischen Musik riskiert eine Musikpublizistik, der es an Distanzierungsvermögen mangelt, zum Bulletin auktorialer Selbstauslegung und zum Stichwortgeber von Komponist*innen zu werden. Das stärkt die Gruppenkohäsion in einem ohnehin hermetischen Kultursegment und trägt damit zu jenen »Diskurs-Wucherungen« (XV) bei, die den Blick auf die Musik selbst verstellen. Für die Herausgeber liegt die Lösung dieses Problems, neben der Absage an Fortschrittsideologie und dichotome Geschichtskonstruktionen, in einem Pluralismus der Zugangsweisen zum Gegenstand - eine Maxime, die keinen Widerspruch zu befürchten hat und die für ein per se 
vielstimmiges Handbuch eine tragfähige $\mathrm{Ar}$ beitsgrundlage abgibt.

Und ein Handbuch ist es ebenso sehr wie ein Lexikon. Dem im engeren Sinne lexikalischen Teil mit insgesamt 104 Artikeln gehen neun teils umfangreiche Darstellungen zu zentralen Themen der neuen Musik voraus. Gemäß den Grundentscheidungen des Bandes sollen sie gerade keine musikgeschichtliche sgroße Erzählung, konstituieren, sondern mit ihrer Aspektvielfalt, die u. a. historische, kompositionstechnische, ästhetische und weltanschauliche Perspektiven umfasst, der tatsächlichen Fragmentierung und Pluralisierung des Gegenstands gerecht werden. Trotzdem beginnt auch hier die Geschichte in Darmstadt, ${ }^{1}$ wenngleich auf erhellende Weise. Denn Ulrich Mosch zeigt in seiner kompakten und sachlichen Darstellung der zentralen Diskussionen der Avantgarde und ihrer Rezeption durch die Musikwissenschaft (3-16), wieviel mehr als geschichtsphilosophische Anmaßung hinter ihren selbstgewissen Stellungnahmen stand. Stichworte wie ,Klangkomposition`, 'Form‘, 'Musik im Raum`, 'Zufallı und auch Adornos Diagnose vom `Altern der Neuen Musikı erscheinen hier als Knotenpunkte einer weitgehend offenen Auseinandersetzung über Grundfragen zeitgenössischen Komponierens und nicht als einbetonierte Streckenposten auf dem Weg in die Zukunft.

An die Epochenorientierung des Beitrags von Mosch schließt derjenige von Christian Utz über "Neue Musik als Klangorganisation « in seiner chronologischen Anlage an (35-53). Als historische Ausformungen des "Komponierens mit 'Klang، " (41) behandelt er neben Edgard Varèse, John Cage und Giacinto Scelsi am einen sowie Gérard Grisey und Luigi Nono am anderen Ende des Zeitraums (und zahlreichen weiteren kurz erwähnten Komponisten und Werken) schwerpunktmäßig György Ligetis Kompositionen der frühen 1960er Jahre. Zum historischen Durchgang anhand der verschiedenen zeitlich aufeinander folgenden Vertreter tritt die Diskursebene hinzu, wenn Utz beispielsweise die früh zu einem musikpublizistischen Topos gewordene Gegenüberstellung von Ligeti und Krzysztof Penderecki (zu Lasten des Letztgenannten) zeitgeschichtlich, unter
Hinweis auf einen »vergleichbaren politischsozialen Erfahrungshintergrund« (43) relativiert oder wenn in der Darstellung von Helmut Lachenmanns Klangtypologie anstelle einfacher Abgrenzung dessen kritische Bindung an die Klangkomposition deutlich wird. Was die Einleitung als Forderung formulierte - dass die Musikgeschichte nach 1950 nicht als Geschichte von Kontroversen und Dichotomien aufgefasst werden solle $(\mathrm{XV})$ - bekommt hier Konturen.

Lukas Haselböcks Beitrag zum mikrotonalen und spektralen Komponieren (103-115) setzt diejenigen von Mosch und Utz zwanglos fort, indem er in einem weitgehend chronologischen Durchgang und trotz des Eingeständnisses ihrer Unmöglichkeit $(104,113)$ eine »Geschichte der Mikrotonalität im 20. und 21. Jh." (113) erzählt. Wie sehr dabei Kontinuitätsvorstellungen unterschwellig weiterwirken, zeigt sein Ansinnen, das historische Material »im Sinne einer ,Gleichzeitigkeit des Ungleichzeitigen « (104) darzustellen. Die Rede von der Ungleichzeitigkeit setzt eben immer ein normatives Wissen um das voraus, was san der Zeit ist‘; sie kann, mit Achim Landwehr zu reden, ihre »Herkunft aus den diversen Sparten der Modernisierungstheorien kaum verbergen und stellt somit eines der letzten Reservate des Eurozentrismus dar. Denn wenn man Ungleichzeitigkeit konstatiert, dann muss man das von einem bestimmten Standpunkt aus tun, und dieser Standpunkt kann wahlweise 'Fortschritt', >Avantgarde`, > Elite oder sonst wie heißen. «"

Interessant wäre darüber hinaus eine eingehendere Betrachtung der jeweiligen Gründe für die Verwendung von Mikrotönen durch die Komponist*innen: Geht es ihnen um die Erweiterung der Materialbasis, gegebenenfalls unter Beibehaltung traditioneller Kompositionsprinzipien? Um eine Ausdifferenzierung der Harmonik? Um eine Flexibilisierung der Melodik als Anpassung an die Flexionen nicht temperierter oder nichteuropäischer Musik? Um Biomorphismen wie in den frühen spektralen Kompositionen? Ein solch systematisierender Zugriff auf den Gegenstand vermiede wohl auch unerwünschte historiographische Implikationen.

2 Landwehr 2012, $19 f$. 
Die Texte von Elena Ungeheuer zur digitalen Musikgestaltung (77-87) und von Christa Brüstle über Raumkomposition und Grenzüberschreitungen zu anderen Künsten (88-102) fügen sich in die latente Chronologie der besprochenen Beiträge ein, ohne sie aber in ähnlich direkter Weise fortzusetzen. Beide berücksichtigen die historische Dimension ihres Gegenstands, lassen ihn aber eher parallel zu einer 'großen Erzählung verlaufen, als ihn darunter zu subsumieren. Zusammen mit Martin Supper zeichnete Elena Ungeheuer 1995 für den Artikel über »Elektroakustische Musik« in der Neuausgabe der MGG verantwortlich. Zwanzig Jahre später können beide (Supper im lexikalischen Teil unter dem Lemma »Elektronische Musik/Elektroakustische Musik/Computermusik", 218-226) aktualisierend und in die Gegenwart fortschreibend an ihre damalige Darstellung anknüpfen. Durch die Verbindung von Medien- und Musiktheorie zur Beschreibung der ästhetischen und kompositorischen Praxen ergeben sich dabei Anschlüsse z. B. an den Beitrag von Ulrich Mosch (vor allem 80f.) oder, neben einzelnen Lemmata des lexikalischen Teils, auf die jeweils verwiesen wird, an den unmittelbar anschließenden Text über Raumkomposition (vor allem Abschnitt 2.5.1, 85f.). Letzterer folgt in der Darstellung seiner Gegenstände einem katalogisierenden Ansatz, um die Fülle und Heterogenität des Materials gerade zu diesem Aspekt erfassen zu können. Neben der (auch bei Mosch schon behandelten) Idee der Raumkomposition in den 1950er Jahren werden Happening und Fluxus ebenso berücksichtigt wie die Klanginstallation und (wie schon bei Ungeheuer) Aspekte der LiveElektronik.

Die beiden Essays von Jörn Peter Hiekel (54-76 und 116-134) scheren aus der vorherrschenden Perspektive aus, indem sie mit Weltund Glaubensbezügen zwei einander ergänzende Spielarten der Referenzialität von Musik in eher systematischem Zugriff zur Sprache bringen. Unter "Weltbezogenheit» versteht Hiekel in erster Linie politisch engagierte Musik, als zentraler Aspekt erscheint ihm dabei die Frage nach der Deutlichkeit der Bezüge (63). Das Gegenbild, vor dem er seinen Gegenstand Profil gewinnen lässt, sind der "weltabgewandte[ ] Materialfortschritt« (66) bzw. ein »eindimensionale[r] Avantgardebegriff« (67). Zu- gleich attestiert er aber auch Kompositionen der seriellen Musik zumindest "subkutane" Weltbezüge (62f.). In der Tat: Historisiert man nur konsequent die Idee eines verbindlichen Materialstands und mit ihr den Begriff der Avantgarde, dann treten Weltbezüge - im Sinne eines Versuchs der Kontingenzbewältigung durch die Reetablierung normativer Kriterien so deutlich zutage, dass es für die defensive Art, in der dieser Gedanke von Hiekel formuliert wird, gar keinen Grund gibt. Diese Vorsicht mag auch Diplomatie sein: Beide Texte scheuen vor Festlegungen zurück und schleifen pointierte Deutungen im Gang ihrer Diskussion gerne wieder ab (z. B. 60, 62). Vielleicht sind sie aus Einleitungsreferaten erwachsen, die alle Positionen würdigen und niemandem zu nahe treten wollen. ${ }^{3}$ Das erklärte dann auch, warum eine Kontroverse wie die um die Rolle des Spirituellen in der Musik der Gegenwart (immerhin seinerzeit ein Tagungsthema des Instituts für Neue Musik und Musikerziehung [INMM] Darmstadt, also wohl eines mit Diskussionspotenzial) vorzeitig mit einem Schillerzitat abgelöscht wird (132) oder warum die zeitgeschichtlich signifikante Auseinandersetzung zwischen Helmut Lachenmann und Hans Werner Henze unter Hinweis auf Parallelen bei Schiller und Fichte ins Überzeitlich-Allgemeinmenschliche gehoben und so neutralisiert wird (66).

Neben der Verabschiedung der großen Geschichtserzählungen liegt ein weiterer Leitgedanke des vorliegenden Lexikons in der Anwendung einer nicht-eurozentrischen Perspektive auf seinen Gegenstand. Dafür stehen neben den Artikeln zu einzelnen Ländern und Regionen vor allem die Themenbeiträge von Wolfgang Rathert zur amerikanischen Musikgeschichte und von Christian Utz zu »Transnationalen Tendenzen neuer Musik seit 1945« (135154). Insbesondere bei Utz kommt der Idee einer Globalgeschichte der Musik einiges Gewicht zu, wenn er auch die Schwierigkeiten ihrer Realisierung klar benennt (137f.). Das methodische Ideal einer histoire croisée, einer »transnationalen musikalischen $>$ Verflechtungsgeschichte« (144), die gleichermaßen lokale

3 Vgl. Hiekel 2015 und 2008. Beide Publikationen werden in den Literaturangaben der jeweiligen Beiträge nicht erwähnt. 
und nationale Diskurse berücksichtigt bzw. von innen quergelesen wird (138), bleibt einstweilen noch uneingelöst, mehr regulative Idee als operationalisierbares methodisches Programm. Schon jetzt aber kann diese Perspektive durch Erkenntnisse überzeugen, die anders nicht zu haben wären - das zeigen die drei Fallbeispiele, die globale Verflechtungen der neuen Musik in Japan, China und Korea in der Zeit des Kalten Krieges offenlegen.

Für die Musikgeschichte Nordamerikas diskutiert Wolfgang Rathert die Frage eines "Sonderwegs", und es ist eine der Stärken seines Beitrags (17-34), dass er sie nicht entscheiden will, sondern aus der ihr zugrunde liegenden Spannung von Exzeptionalismus und Universalismus Leitlinien seiner Darstellung gewinnt. Wenn dabei berücksichtigt werden muss, dass die »besonderen historischen Voraussetzungen der amerikanischen Musikkultur [...] weniger mit ästhetischen Konzepten zu tun [haben] als mit gesellschaftlichen und kulturellen Konstellationen, in die Musik stark involviert ist« (20), fügt dies den vielen Perspektiven des Bandes noch eine weitere, dezidiert geschichtswissenschaftliche hinzu. ${ }^{4}$ Die Vorstellung einer relativen Autonomie der Musikgeschichte ist unter solchen Voraussetzungen weniger denn je zu halten. Dementsprechend gehen die zahlreichen Erkenntnisse des Beitrags stets auch mit allgemeinhistorischen Perspektiven einher wenn etwa an der Liste der Pulitzer-Preisträger nicht nur die Entwicklung der amerikanischen musikalischen Moderne abgelesen wird, sondern auch sozialhistorische Aspekte der spezifisch amerikanischen Figur des »Maverick"
(22), oder wenn die Aneignung der amerikanischen Avantgarde durch die europäische Linke in den späten 1960er Jahren als geschichtsphilosophisches misreading einer ihrerseits bereits politisch eminent aufgeladenen Kunst herausgestellt wird (32f.).

Ratherts Ausführungen im Themenbeitrag lassen sich sinnvoll mit dem ebenfalls von ihm verfassten Artikel »Nordamerika" (464-469) zusammenlesen. Aber auch dann, wenn Themen- und Lexikonbeiträge von verschiedenen Autor*innen stammen, bieten sich durch die besondere Anlage des Bandes zugleich als Handbuch und Nachschlagewerk willkommene Ergänzungs-, Vertiefungs- und Abschweifungsmöglichkeiten. Das Verweissystem ist präzise genug, um im Rahmen einer schnellen Informationssuche nützlich zu sein, und gleichzeitig so großzügig gehandhabt, dass man sich von ihm (und der eigenen Neugier) auch forttragen lassen kann. Über Entscheidungen wie die Auswahl der Lemmata und ihre Gewichtung oder über den inhaltlichen Zuschnitt einzelner Artikel kann man als Rezensent leicht einmal anderer Meinung sein; auch ließe sich kritisieren, dass manche Artikel sich zu sehr auf ein Literaturreferat beschränken und Bemühungen um eine konzise Synthese vermissen lassen. Aber entscheidend ist das nicht. In der Praxis hat sich das Buch bereits bewährt, und dass es auch außerhalb des Fachs, von Historiker*innen zum Beispiel, hochgeschätzt wird, stellt ihm ein glänzendes Zeugnis aus.

Markus Böggemann
4 Mittlerweile ist Ratherts gewichtige Gesamtdarstellung zum Thema erschienen, vgl. Rathert/Ostendorf 2018. Es bestätigt die hier hervorgehobenen Qualitäten seines Ansatzes, dass das Buch zusammen mit einem Amerikanisten verfasst wurde. 


\section{Literatur}

Hiekel, Jörn Peter (2008), »Sinnstiftung und sakrale Sehnsüchte. Überlegungen zum Aspekt des Spirituellen in der Musik«, in: Sinnbildungen. Spirituelle Dimensionen in der Musik heute, hg. von Jörn Peter Hiekel, Mainz: Schott, 9-21.

Hiekel, Jörn Peter (2015), »Randständig oder zentral? Welt- und Gegenwartbezüge in Musik«, in: Zurück zur Gegenwart? Weltbezüge in neuer Musik, hg. von Jörn Peter Hiekel, Mainz: Schott, 10-31.
Landwehr, Achim (2012), »Von der >Gleichzeitigkeit des Ungleichzeitigen«", Historische Zeitschrift 295/1, 1-34.

Rathert, Wolfgang / Berndt Ostendorf (2018), Musik der USA. Kultur- und musikgeschichtliche Streifzüge, Hofheim: Wolke.

Rebstock, Matthias (2017), „Paradoxien zwischen Aneignung und Abgrenzung. Überlegungen zur kulturellen Identität der neuen Musik«, Positionen Heft 111, 3-8.

Böggemann, Markus (2019): Jörn Peter Hiekel / Christian Utz (Hg.), Lexikon Neue Musik, Stuttgart: Metzler / Kassel: Bärenreiter 2016. ZGMTH 16/2, 187-191.

https://doi.org/10.31751/1021

(C) 2019 Markus Böggemann (boeggemann@uni-kassel.de)

Universität Kassel [University of Kassel]

Dieser Text erscheint im Open Access und ist lizenziert unter einer

Creative Commons Namensnennung 4.0 International Lizenz.

This is an open access article licensed under a

Creative Commons Attribution 4.0 International License.

eingereicht / submitted: 09/11/2019

angenommen / accepted: 12/11/2019

veröffentlicht / first published: 23/12/2019

zuletzt geändert / last updated: 10/01/2020 